\title{
Animal Hygiene and Animal Health in Dairy Cattle Operations
}

\author{
Jos P. Noordhuizen * and Joao Cannas da Silva
}

Dip. Eur. College Bovine Health Management, VACQA-International, Santarém, Portugal

\begin{abstract}
This paper addresses the economic and strategic position of animal health in the dairy operations and the way that animal health care should be properly designed. Examples of biosecurity plans and quality risk management are further elaborated. Disease risk identification and disease risk management as primary preventive issues are pivotal in modern animal health care on both the small and larger dairy farms. Biosecurity and quality risk management can both be integrated into current operational veterinary herd health and production management programs.
\end{abstract}

Keywords: Dairy cattle, animal hygiene, animal health, biosecurity, quality risk management.

\section{INTRODUCTION}

In all dairy operations, small or large, animal health care represents a major component, next to genetics, feeding and management quality. Firstly, because healthy animals can produce milk more efficiently and healthy calves can grow in a more optimal way. Secondly, because healthy animals will show a better feed intake pattern throughout lactation as well as a better fertility performance. Thirdly, because healthy animals will show less welfare problems throughout their life, hence, improving herd longevity [1]. Fourthly, because animal health status can have a large impact on public health and food safety issues. Health is, basically, a feature of the dairy production process quality.

Animal health care, comprising both non-infectious and infectious diseases, can be focused on prevention and eradication (epidemic diseases), on vaccination strategies, as well as on disease reduction and control of (often endemic) diseases by either a curative approach or a risk identification and risk management approach. Disease risk management strategies, addressing several animal hygiene issues, have not been widely adopted yet. Within the $\mathrm{EU}$, disease risk management becomes however more relevant because the use of antibiotics has been more restricted or better justified while the use of anti-microbial feed additives was banned.

Another issue regarding the animal health care concerns the economic issue. It is well-known that curing diseases is costly, the more because the disease losses have already occurred. On the other hand, preventive animal health care costs are hardly or not seen as an investment; this represents a knowledge, perception and communication problem [2].

Proper animal health care takes into consideration not only the veterinary-technical and zootechnical issues, but also the microbiological and epidemiological disease aspects, managerial and economic relevance, and furthermore product and production process quality assurance related features. As an ultimate outcome of such exercises, veterinary herd health $\&$ production management programs may

*Address correspondence to this author at the Dip. Eur. College Bovine Health Management, VACQA-International, Santarém, Portugal;

E-mail: jos.noordhuizen@orange.fr be designed, as well as biosecurity plans, and/or quality risk control programs $[3,4]$.

In this paper the forenamed items will be addressed and discussed in more detail ${ }^{1}$.

\section{VETERINARY-AND ZOOTECHNICAL ISSUES}

Health care or disease combat is classically characterized by two approaches:

1. eradication and prevention, which predominantly regards epidemics of OIE list A and list B diseases like foot-and-mouth-disease, brucellosis, BSE, leptospirosis. The art is in designing and implementing proper biosecurity plans to keep these diseases out of a country and of a farm. And in case such a disease has entered a country the legal procedures of eradication will be started.

2. reduction and control, which regard the mostly endemic infectious diseases of OIE list $\mathrm{C}$ and $\mathrm{D}$, like BVD, IBR, and mastitis, and non-infectious diseases like lameness, ketosis, fertility disorders. In the case of infectious diseases among those, one may consider vaccination programs (if allowed by e.g. the EU), vaccination $\&$ following test-cull strategies like for IBR in the EU. In the latter situation, markervaccination is used for first reducing the prevalence of a disease in a region or country, while the disease is subsequently eradicated sector-wide through e.g. a test-and-cull-policy.

In both approaches the appropriate diagnostic tools should be available, with known sufficient sensitivity and specificity for the proper application in different disease situations.

In case of vaccinations, distinction should be made between the two forenamed approaches, because during epidemics usually vaccination is not allowed, or only to limit such a disease geographically in order to facilitate eradication later on, while in endemic situations vaccination is not (always) allowed by a sector (for reasons of trade in Europe

\footnotetext{
${ }^{1}$ This paper is a reflection of an oral presentation given at the ISAH congress in Warsaw, Poland, in 2005.
} 
for example), not available, or not sufficiently effective in a given situation to reduce or control such diseases. In epidemics there is a trend towards the input of deletion vaccines with adjacent discriminative diagnostics, which might facilitate their introduction in campaigns.

Endemic diseases such as mastitis and lameness are very often multi-factorial in nature, meaning that different contributory factors ultimately leading to disease can be involved. Such contributory factors may be found at animallevel, such as age/parity, milk yield level, or lactation stage, or the cow-environment-level, such as housing conditions, feeding related features, or climatic conditions. The forenamed so-called risk conditions can be identified through monitoring plans, their impact assessed by adaptive conjoint analysis procedures involving experts [5] or quantified by epidemiological studies yielding odds ratios or relative risks [6].

Table 1. An Example of Some Risk Conditions for Listeriosis [7] and S. aureus Mastitis [8] in Dairy Cows Respectively; A Qualitative Approach. $\mathrm{SA}=S$. aureus; $\mathrm{SU}=$ Strep uberis

\begin{tabular}{|c|c|}
\hline Listeriosis & S. aureus mastitis \\
\hline \hline Low silage $\mathrm{pH}$ & Old parity cows \\
\hline Dirty housing and cows & Previous udder infection by SA/SU \\
\hline Poor bedding quality & Poor milking hygiene \\
\hline Wet udder preparation & Poor teat end callosity scores \\
\hline Milking machine failures & Milking machine failures \\
\hline Poor cleaning \& disinfection & Number of quarters involved \\
\hline
\end{tabular}

Risk conditions can be found at the animal/herd level (e.g. parity; milk yield; breed; lactation stage), the level of cows' environment and management (e.g. barn climate; housing conditions; feed quality), and farm information (milk recording; feedstuff analysis). In Table $\mathbf{1}$ an example is given of an identification of risk conditions for two infections, while in Table $\mathbf{2}$ an example is given of risk quantification originating from a population study [6].

\section{MICROBIOLOGICAL ISSUES}

With regard to animal health care it is well-known that different infectious agents have different characteristics regarding virulence and pathogenicity, transmission routes, survival in the environment, susceptibility for antibiotics. These features are relevant to understand when working to design an animal health care plan for dairy farms. In Table $\mathbf{3}$ a few of these features have been highlighted for several infectious agents [9].

\section{MANAGERIAL AND ECONOMIC ISSUES}

Animal health care is by excellence a management issue. Management has to balance the inputs as well as control the risk conditions on the farm in order to achieve an optimal income. This refers to decision-making under uncertainty. Animal health care puts demands on the farmer with regard to the routine monitoring of farm conditions and animals concerning signs of pending mal-performance and early diagnostics of a disease in its development stages. Further- more, the farmer takes care of treatment follow-up once a treatment to a cow is given, as well as of the appropriate moment for restarting delivery of milk (withdrawal period). In the discussion between farmer and veterinarian, the choice of drugs will be put forward, a costs assessment will be provided, including a prognosis as well as the desired duration of treatment, given a microbiological and clinical recovery.

Table 2. An Example of Quantified Risk Conditions Using the Odds Ratio as Read Out Parameter: The Case of Mortellaro Disease in Dairy Cattle [6]

\begin{tabular}{|lll|}
\hline Risk Factor of Concern & Clsss & Odds Ratio \\
\hline \hline Parity & 1 & 1.32 \\
& 2 & 1.05 \\
& 3 & 1.0 (reference value) \\
\hline Stage of 1 & Dry & 0.34 \\
actation & Pre-top & 0.81 \\
& Top & \\
& (50-70 days) & 1.70 \\
& Past top & 1.0 (reference value) \\
\hline Access to & Limited & 1.51 \\
pasture & Free & 1.0 (reference value) \\
\hline Walking & $>200 \mathrm{~m}$ & 5.37 \\
distance & $<200 \mathrm{~m}$ & 1.0 (reference value) \\
\hline Walking & Metalled & 2.56 \\
path & Grass & 1.0 (reference value) \\
condition & & \\
\hline Odds ratio > 1 means risk increase; Odds ratio < 1 means risk decrease.
\end{tabular}

Disease and disease control are economic issues, as illustrated in Fig. (1), adapted after [2].

Disease control means less production loss and costs, more farm income [2].

There is not only a wide variation between dairy farms with regard to the prevalence of several diseases, but also with regard to the risk conditions prevailing on those farms. At least partly, this variation is associated with the observational skills of a farmer to detect early signs of disease, his awareness about risk conditions on his farm and the knowledge to deal with those risk conditions properly, his risk avoidance or risk prone attitude related to health care, and his decision making practice in economic matters.

Risk identification and risk management strategies have become a major relevance in the dairy sector these days. This is -as stated before- partly caused by the non-vaccination policy in the EU, but even more by the severe disease outbreaks over the last decades which dramatically shuttered the farming community.

\section{BIOSECURITY PLANS}

Biosecurity Plans are meant to control and avoid the introduction of infectious agents into as well as the spread of such agents on the premises of the farm. Biosecurity plans refer to health management strategies and comprise key components like formal disease risk identification and risk assessment on a particular farm [9]. These plans make proper use of the issues addressed in forenamed paragraphs and convert these into a set of so-called working instructions or protocols. For example, a Protocol on General Hygiene pro- 
Table 3. Differences in Transmission Routes and Environmental Survival of Some Bovine Pathogens [9]

\begin{tabular}{|l|c|c|c|}
\hline & S. aureus & Johne's & BVD \\
\hline \hline Transmission routes: & & + & \\
$\quad$ Faecal - oral & & & + \\
Nasal/saliva & $?$ & + & + \\
Milk-borne & + & + & + \\
In utero & & & + \\
Sexual & & mo - yrs & $10-14$ days \\
\hline Shedding time: & Days - years & mo - yrs & $<14$ days \\
\hline Environmental survival: & $?$ & wks-mo \\
\hline Environmental growth: & yes? & mo \\
\hline
\end{tabular}

$?=$ unknown; += yes; no symbol presented $=$ no.

mo $=$ month; wks $=$ weeks.
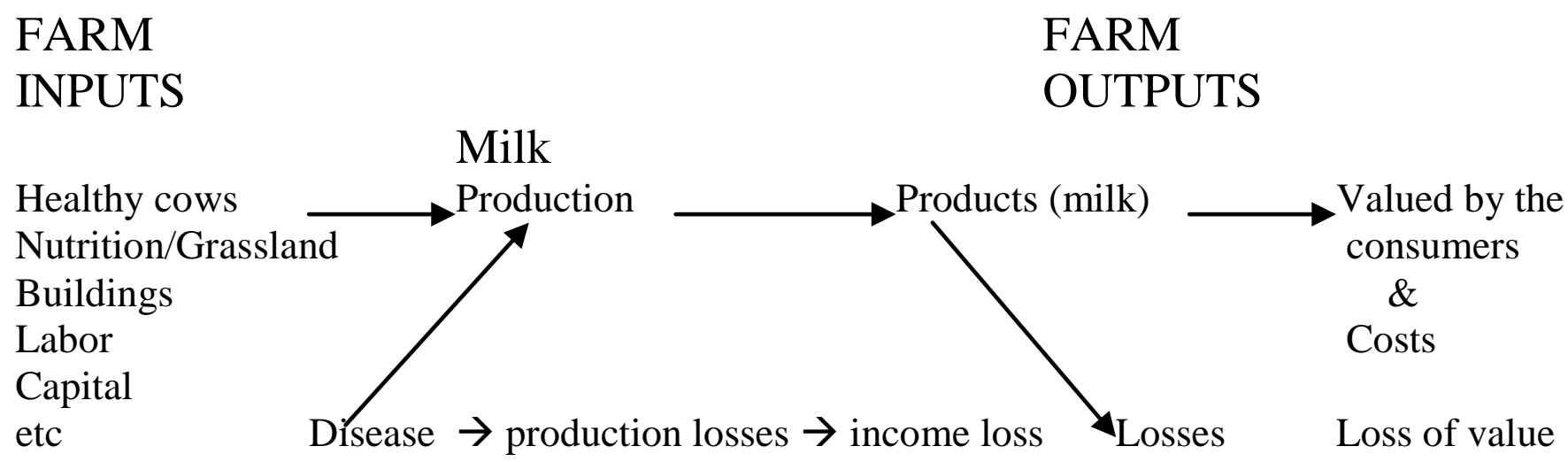

Fig. (1). An illustration pointing to disease as an economic issue at both farm and consumer level.

cedures, a Protocol on Entrance Procedures for animals, cars, professionals, cattle, a Protocol on Disease diagnostics \& Animal treatment, or a Protocol on Good Medicine Application Practice.

Basically these protocols are tailor-made working instructions for the farmer and his farm-workers. The main objective is to reduce the effects of risk conditions or control these risks so that probability of disease will be as low as reasonably feasible on that particular dairy farm. Animal hygiene issues are essential elements in a biosecurity plan. Biosecurity is focused on both disease prevention and disease risk control. These plans are only functional when the proper hygiene practices prevail on a dairy farm, and when all who work on or around the farm have adopted the rules set out. The working instructions must therefore be discussed with all farm workers and advisors; when needed specific instruction courses must be given on-site. In fact, biosecurity builds on further on a general good farming practice attitude.

When the most relevant health hazards have been identified on a farm, the associated risk conditions for both introduction and spread of infections have to be identified. This can be done by conducting formal epidemiological studies, by searching text books for risk factor profiles (see Table 1), or by applying expert interviews e.g. adaptive conjoint analysis [5]. The risk factors can then be quantitatively ranked in order of relevance. Then risk weighting (probabil- ity $\mathrm{x}$ impact) takes place to determine whether the risk is probable and or has a high impact. This can be considered paramount for designing risk prevention, -elimination and/or -reduction in biosecurity plans.

For introducing infectious diseases onto a farm the following items are relevant risk conditions: purchasing new cattle from unknown sources, transportation trucks, mixing of cattle with different health status, crossings of dirty and clean roads on the farm, professional visitors not complying to hygiene protocols, lack of quarantine, manure spread, wildlife contacts, pet animals, and rodents.

In Table 4 an example of the build up of biosecurity plans is presented, once the hazard identification and risk assessment have been conducted.

\section{QUALITY RISK MANAGEMENT ISSUES}

Quality on livestock farms usually refers to both the product (milk; beef; live animals) and the production process. Animal health, animal welfare and public health/food safety are features of the production process on a dairy farm. Different quality assurance systems for livestock address different areas: some address the quality of milk only with a particular focus on residues of antibiotics, others address the farmer's attitude regarding quality of the end-product by applying Good Farming Practice, GFP, guidelines, and again others address many different farming areas in an integrated 
manner. The Dutch KKM (Chain Quality Dairy) is an example of the latter approach where both animal health, animal welfare, waste management \& environment, and medicine use are addressed [10].

\section{Table 4. The Build Up of a Biosecurity Plan in 10 Steps}

1. Make the farm more closed (canals; fences; ditches; natural vegetation)

2. Design a People Entrance Protocol (hygiene barrier; boots; clothes)

3. Design an Animal Entry/Exit Protocol (health status' certificates; vaccinations; trucks)

4. Determine on-farm Units and Sections; design Protocols for each unit/section

5. Set protocols for Health Care, for Feeding Management; Hygiene \& Disinfection

6. Design an on-farm Monitoring Protocol

7. Instruct Farm workers; assign responsibilities; evaluate performances

8. Define a farm-specific Training Program for the farm workers in the different units

9. Evaluate the critical risk conditions in all units on fixed time points

10. Have regular Team Assessments for possible adjustments of the biosecurity plan

Product quality (milk) has a long-standing history: somatic cell count, bacteria count, residues, freeze-point determination, chemical and microbiological contamination. For dairy farmers it has always been a matter of brinkmanship's honor to deliver first class milk, meaning that no penalties had been given.

This is already part of a GFP standard. But GFP guidelines should comprise more than this: they should address general risk conditions which contribute to disease occurrence as a general farmer's professional behavior on his farm. An example is the Hygiene Room Protocol at the entrance of the farm: you have to change clothes and boots, and wash your hands.

Production process quality means that the different farming areas should be identified, and within each area the potential health hazards identified as well as their associated risk conditions. As stated before, hazards may be in the area of animal health, but also animal welfare and public health. If animal health is considered a quality feature [11], then quality risks refer to health risks, and quality failure losses to diseases losses. In quality control concepts not all risk conditions are addressed but focus is on the most relevant and feasible ones. Examples are critical control points, $C C P$, which have to comply to 5 formal criteria, while other highly relevant risk conditions not complying to such criteria can be named critical management points or points of particular attention, POPA. The different CCP's and POPA's can subsequently be put together in an on-farm monitoring system. Examples of CCP and POPA are given in Table 5. Such a system focuses on the monitoring points, the responsible farm worker, the frequency of monitoring, and the recording of findings (including those from a laboratory), and the overall evaluation of the integrated quality control.
Table 5. Some Hazard Areas and their CCP or POPA on a Dairy Farm, Examples

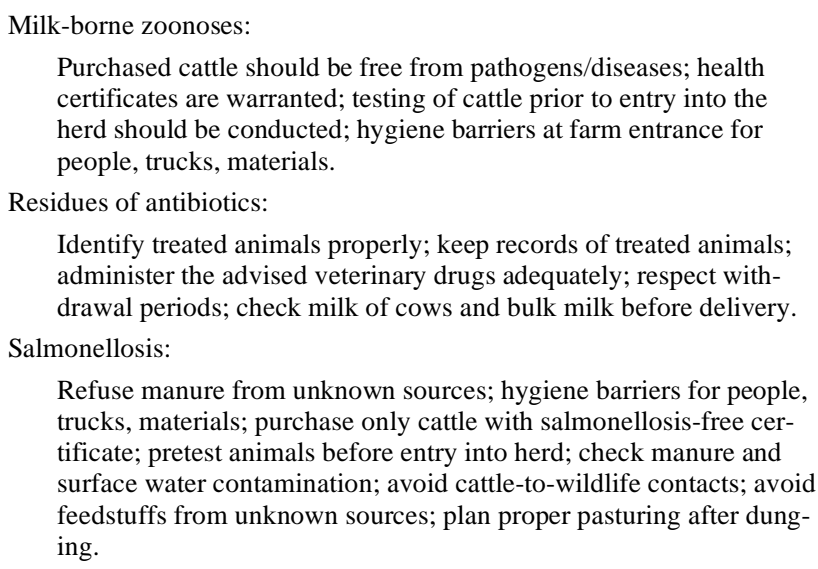
certificates are warranted; testing of cattle prior to entry into the herd should be conducted; hygiene barriers at farm entrance for people, trucks, materials.

Residues of antibiotics:

Identify treated animals properly; keep records of treated animals; administer the advised veterinary drugs adequately; respect withdrawal periods; check milk of cows and bulk milk before delivery.

Salmonellosis:

Refuse manure from unknown sources; hygiene barriers for people, trucks, materials; purchase only cattle with salmonellosis-free certificate; pretest animals before entry into herd; check manure and surface water contamination; avoid cattle-to-wildlife contacts; avoid feedstuffs from unknown sources; plan proper pasturing after dunging.

An example of a quality risk management program as meant above is the concept of hazard analysis critical control points, HACCP. Key elements of this concept are hazard identification, risk identification, risk assessment, definition of CCP's and POPA's in a monitoring program, protocols or working sheets and instructions for correcting possible lost control, and validation [12].

Currently, several countries are active in developing HACCP-like programs for application on dairy farms, and comprising elements like animal health, animal welfare, and food safety.

An example of a part of such a HACCP-like handbook part is presented in Table $\mathbf{6}$.

\section{CONCLUDING REMARKS}

The different elements discussed in this paper have been built up from solitary elements gradually into operational herd health programs [1], biosecurity plans [9] and, ultimately, more tactical quality risk management programs $[3$, 4, 10-12] in an integrative manner.

Recently issued EU directives like the new Hygiene directives (852-853-854 - 2004) and regulation 178-2002 point to a new strategy regarding consumer protection and food safety. Public health and food safety are leading issues, while animal health and welfare are secondary. Nevertheless, it has been stated that the implementation of HACCPcompatible programs may become compulsory for animal health, welfare and food safety on dairy farms and pig farms. The rationale is that farmers have to demonstrate their herd status in the three areas named as well as the actions they take to maintain or improve that status, in order to retain their license to produce and to market their products. This means that around 2015 that implementation should have been achieved. A look at Tables $\mathbf{5}$ and $\mathbf{6}$ will show that most of the issues raised there are not new; what is new that these issues are far more structured and that the approach has been formalized. These both features would highly facilitate certification procedures on dairy farms. 
Table 6. A Part of a HACCP-Like Program for a Dairy Farm: The Components of Cow Treatment in a Lactating Cows' Barn and Milking Parlor [4]

\begin{tabular}{|c|c|c|c|l|}
\hline Hazard (Type) & Risk Weighed Probability x Impact & True Risk? & CCP POPA & \multicolumn{1}{|c|}{ Corrective and Preventive Measures } \\
\hline \hline Wrong drug used & $2 \times 2=4$ & No & POPA & $\begin{array}{l}\text { Set proper diagnosis. Check drug label; see Work } \\
\text { Sheet TAP }\end{array}$ \\
\hline Wrong dosage applied & $2 \times 2=4$ & No & POPA & as above \\
\hline Off-shelf-life drugs & $2 \times 1=2$ & No & POPA & $\begin{array}{l}\text { Check expiration date. } \\
\text { See Work Sheet TAP. }\end{array}$ \\
\hline $\begin{array}{c}\text { Wrong cow-ID during } \\
\text { withdrawal period }\end{array}$ & $2 \times 3=6$ & Yes & CCP & $\begin{array}{l}\text { Apply proper cow-ID } \\
\text { Stick to withdrawal. } \\
\text { See Work Sheet TAP }\end{array}$ \\
\hline Antibiotics residues & $2 \times 3$ & Yes & CCP & as forenamed \\
\hline S. aureus in milk & $3 \times 2$ & Yes & CCP & $\begin{array}{l}\text { Apply udder health control program. } \\
\text { Deliver no mastitis milk. }\end{array}$ \\
\hline
\end{tabular}

$\mathrm{TAP}=$ treatment advisory plan, as designed by the veterinarian every 6 months.

$\mathrm{ID}=$ identification; $\mathrm{CCP}=$ critical control point; $\mathrm{POPA}=$ point of particular attention.

\section{REFERENCES}

[1] Brand A, Schukken YH, Noordhuizen JPTM. Veterinary herd health and production management in dairy practice. Wageningen Academic Publishers, Wageningen: The Netherlands 1996; p. 543.

[2] MacInerney JP. The economic analysis of livestock diseases: a developing framework. Acta Vet Scand Suppl 1988; 84: 66-74.

[3] Noordhuizen JPTM. HACCP, total quality management and dairy herd health. In: Roginski H, Fuquay JW, Fox PF, Eds. Encyclopedia of Dairy Sciences. Academic Press: London 2003; vol. 3: pp. 1281-9.

[4] Lievaart JJ, Noordhuizen JPTM, van Beek E, et al. The hazard analysis critical control point concept as applied to some chemical, physical and microbiological contaminants of milk on dairy farms, a prototype. Vet Q 2005; 27: 21-9.

[5] Horst HS, Huirne RBM, Dijkhuizen AA. Eliciting the relative importance of risk factors concerning contagious animal diseases using conjoint analysis: a preliminary report. Prev Vet Med 1996; 27: 183-95.

[6] Noordhuizen JPTM, Frankena K, Thrusfield MV, et al. Application of quantitative methods in veterinary epidemiology. $2^{\text {nd }}$ ed. Wagen- ingen Academic Publushers: Wageningen, The Netherlands 2001; p. 429.

[7] Sanaa M, Poutrel B, Menard JL, et al. Risk factors associated with contamination of raw milk by Listeria monocytogenes in dairy farms. J Dairy Sci 1993; 76: 2891-8.

[8] Zadoks RN, Allore HG, Barkema HW, et al. Quarter and cow level risk factors for strep uberis and staph aureus mastitis. J Dairy Sci 2001; 84: 2649-63.

[9] BAMN. An introduction to infectious disease control on dairy farms (biosecurity) \& biosecurity on dairies. Bovine Alliance on Management \& Nutrition, Arlington, USA, 2000.

[10] Noordhuizen JPTM, Lievaart JJ. Veterinary quality management: the Dutch total touch. In: Madec F, Clement G, Eds. Proceedings in Congress of the International Society for Animal Hygiene, St Malo (F), October 11-13, 2004; vol. 2: pp. 503-5.

[11] Noordhuizen JPTM, Welpelo HJ. Sustainable improvement of animal health care by systematic quality risk management according to the HACCP concept. Vet Q 1996; 18: 33-7.

[12] Noordhuizen JPTM, Cannas da Silva J, Boersema JSC, et al. Applying HACCP-based quality risk management on dairy farms. Wageningen Academic Publishers, Wageningen: The Netherlands 2008; p. 311.

(C) Noordhuizen and da Silva; Licensee Bentham Open.

This is an open access article licensed under the terms of the Creative Commons Attribution Non-Commercial License (http://creativecommons.org/licenses/by$\mathrm{nc} / 3.0 /$ ) which permits unrestricted, non-commercial use, distribution and reproduction in any medium, provided the work is properly cited. 